\title{
LOS VALORES CULTURALES DE LOS MURCIÉLAGOS
}

\author{
Oscar G. Retana-Guiascón ${ }^{1}$ y María L. Navarijo-Ornelas ${ }^{2}$ \\ ${ }^{1}$ Centro de Estudios de Desarrollo Sustentable. Universidad \\ Autónoma de Campeche. Av. Agustín Melgar s/n Colonia Buenavis- \\ ta. San Francisco de Campeche, Campeche, México 24039. \\ ${ }^{2}$ Departamento de Zoología, Instituto de Biología, Universidad Na- \\ cional Autónoma de México. México, D.F. 04510. \\ Autor de correspondencia: Oscar Retana, ogretana @ uacam.mx
}

\begin{abstract}
RESUMEN
Los murciélagos al igual que otros grupos animales han formado parte del repertorio de percepciones, conceptos y símbolos entre las distintas sociedades mesoamericanas. En el México prehispánico la adhesión de estos vínculos y su transmisión de generación en generación forjaron en la colectividad una serie de valores culturales, los cuales, además de dar cohesión e identidad comunal, han funcionado como elementos de orden y control social, pues de manera directa o indirecta intervinieron en el proceso de conocimiento y uso de la fauna silvestre. Con base en estos conceptos, el objetivo del presente trabajo fue establecer los tipos de valores culturales atribuidos a los murciélagos en el México prehispánico. Por medio de una investigación documental aplicando las técnicas de fuentes secundarias y terciarias, se establecieron cuatro tipos de valores culturales para los murciélagos: iconográfico, religioso; simbólico y mítico, los cuales concentran 15 subtipos de valores, entre los que destaca el vínculo de murciélago como glifo emblema de autoridades, signo calendárico, deidad y elemento totémico. La importancia de este tipo de estudios reside en evidenciar primariamente los valores culturales vinculados a los quirópteros y ubicar su utilidad actual en las estrategias locales y/o regionales relativas a su conocimiento y conservación.
\end{abstract}

Palabras clave: fauna silvestre, valores culturales, conservación, sociedades mesoamericanas

\begin{abstract}
Bats as well as other animal groups have formed part of the traditional knowledge of perceptions, concepts and symbols among the different Mesoamerican societies. In the prehispanic México accession of these links and its transmission from generation to generation forged in the community a series of cultural values, which, in addition to cohesion and communal identity, have functioned as elements of order and social control, thus directly or indirectly involved in the process of knowledge and use of wildlife. Based on these aspects, the objective of the present work was to establish the types of cultural values attributed to bats in the prehispanic México. Through a documentary research applying the techniques of secondary and tertiary sources, establishes four types of cultural values for bats: iconographic, religious; symbolic and mythical, which concentrated 15 subtypes of values, among them the link of bat as glyph emblem of authorities, printed, deity and totemic element sign. The importance of this type of study is primarily evidenced cultural values linked to the bats and locates your current utility on the local and/or regional strategies concerning their knowledge and conservation.
\end{abstract}

Keywords: Bats, cultural values, conservation, mesoamerican societies 


\section{INTRODUCCIÓN}

México es uno de los países que cuenta con un número considerable de especies nativas de mamíferos, se han registrado aproximadamente 529 especies (Ceballos et al., 2005), cantidad que esta apenas por debajo de lo que posee Indonesia (560 especies) o Brasil (540 especies). Los murciélagos no son la excepción, dado que se han reconocido en México cerca de 138 especies (Medellín et al., 2008) de un total estimado de 1,116 en el mundo (Simmons, 2005), que equivale a $12.4 \%$ de la diversidad total. La importancia de estas cifras radica en evidenciar el hecho de que en México la distribución de los quirópteros incluye una gran variedad de hábitats y por tanto los hábitos de las especies son heterogéneos, registrando una mayor riqueza en los trópicos y especialmente en los estados de Oaxaca y Chiapas (Castro y Galindo, 2009). Cabe destacar que en el territorio integrado por Estados Unidos y Canadá solo se distribuye el $5 \%$ de las especies de murciélagos del mundo (Wilson y Reeder, 2005; Eguiarte, 2006), lo cual constituye otra referencia comparativa que ratifica la importancia de este grupo en nuestro país.

Considerando que los murciélagos se distribuyen mundialmente y la mayoría de las especies (microquirópteros) tienen su mayor actividad en las horas crepusculares y a lo largo de la noche, aunado a su aspecto enigmático y refugiarse durante el día en sitios oscuros, son elementos suficientes para que entre las distintas sociedades se crearan en su entorno diversas percepciones, conceptos y simbolismos. Entre los pueblos europeos medievales se posiciono al murciélago como un arquetipo del mal o encarnación del demonio, gestándose mayormente fábulas de horror y temor a estos mamíferos voladores (Castro y Galindo, 2009). Influencia que llegaría al continente americano durante la época colonial y que generaría el establecimiento de diversas leyendas vinculadas en su mayoría a la brujería y el vampirismo.

En el México precolombino, la riqueza y abundancia de los quirópteros, así como su carácter nocturno y el hecho de habitar en lugares como las cuevas, contribuyeron a justificar el hecho de que se originaran diferentes asociaciones simbólicas; en donde; el murciélago se vínculo con el útero, el inframundo, la muerte y con la oscuridad (Muñoz, 2006). Por lo tanto, en el proceso cognoscitivo del mundo natural, los quirópteros, al igual que otros animales formaron parte de la cosmovisión de distintas sociedades mesoamericanas como mecanismos de entendimiento y conexión entre el mundo natural y espiritual. En el México prehispánico el asentimiento de estos lazos de una generación a otra forjó en la colectividad una serie de valores culturales (mítico, sagrado, religioso, mágico, iconográfico, agorero), los cuales fueron utilizados para dar cohesión e identidad comunal, explicar fenómenos naturales, así como afirmar la existencia misma del hombre y su pertenencia a la naturaleza (Sarukhán y Dirzo, 1992; Navarijo, 1999; Retana, 2006).

Los valores culturales son el producto de las relaciones del hombre con la naturaleza y forman parte del sistema cognitivo de un grupo étnico. En otras palabras, la creación, difusión y continuidad de los valores culturales atribuidos a ciertas plantas y/o animales constituyen elementos de orden y juicio en relación a su conocimiento, uso y conservación (Toledo, 1991; La Duke, 1994; UNESCO, 1999).

Basándonos en los planteamientos anteriores, el objetivo general del presente trabajo fue documentar y analizar la atribución de los valores culturales otorgados a los murciélagos por las sociedades prehispánicas de México y su posible referencial entre comunidades indígenas actuales.

\section{MÉTODOS}

Este estudio bibliográfico se fundamenta en la documentación y sistematización de los informes relevantes sobre los valores culturales tangibles e intangibles referidos para los murciélagos, considerando en particular el periodo prehispánico de México. Para obtener los elementos de información y análisis relacionados con el objeto de estudio se condujo una investigación documental aplicando la técnica de fuentes secundarias y terciarias (Tenorio, 1988). Específicamente se efectuó una revisión 
de la literatura impresa y en formato digital relativa a diferentes aspectos culturales en los cuales los murciélagos han sido partícipes.

\section{RESULTADOS}

Conforme a los datos recabados se proponen cuatro tipos de valores culturales vinculados a los murciélagos: 1) iconográfico, 2) religioso, 3) simbólico y 4) mítico, los cuales concentran 15 subtipos de valores (Cuadro 1).

\section{Valores iconográficos}

En el México prehispánico el murciélago fue considerado un ser del inframundo y se le asoció a la decapitación. Asimismo, representó la oscuridad, la tierra y la muerte, sin embargo, también se le asoció con el culto al maíz y la fertilidad (Schlesinger, 2001). No obstante, en el contexto iconográfico se documenta que la figura del murciélago fue utilizada como glifo emblema de autoridades y gobernantes como en el caso de Tecuanapan en el estado de Guerrero o en la región de Calakmul, Campeche (Vega, 1991; Grube, 2005), así como para referir entidades sociopolíticas como Zinacantepec, Estado de México (de: Tzinacan; "murciélago" y Tépetl, "cerro"), representado en el Códice Mendocino por el glifo de un cerro con un murciélago en su cima (Macazaga, 1979). De igual forma, el poblado de Zinacantán en el

Cuadro 1. Tipología de los valores culturales vinculados a los murciélagos

\begin{tabular}{|c|c|c|}
\hline Tipo & Subtipo & $\begin{array}{l}\text { Impacto en las estrategias locales } \\
\text { de conservación }\end{array}$ \\
\hline 1. Iconográfico & $\begin{array}{l}\text { 1.1. Glifo emblema de autoridades. } \\
\text { 1.2. Topónimo de entidades } \\
\text { sociopolíticas. } \\
\text { 1.3. Signo de carácter calendárico. }\end{array}$ & $\begin{array}{l}\text { Se respeta a los murciélagos al ser un } \\
\text { elemento que da cohesión e identidad } \\
\text { cultural. }\end{array}$ \\
\hline 2. Religioso & $\begin{array}{l}\text { 2.1. Totémico, protector de la } \\
\text { comunidad. } \\
\text { 2.2. Divinidad y/o alter ego zoomorfo } \\
\text { de deidades mesoamericanas. } \\
\text { 2.3. Entidad totémica. } \\
\text { 2.4. Sacralización del murciélago en } \\
\text { templos para rituales de iniciación y } \\
\text { purificación. }\end{array}$ & $\begin{array}{l}\text { Al ser un objeto de culto, había } \\
\text { veneración hacia los murciélagos, por } \\
\text { lo que se evitaba alterar los hábitats } \\
\text { en los que se refugiaban. }\end{array}$ \\
\hline 3. Simbólico & $\begin{array}{l}\text { 3.1. Símbolo de fertilidad, relacionado } \\
\text { con la menstruación y la capacidad } \\
\text { reproductiva de la mujer. } \\
\text { 3.2. También se asocia a la fertilidad de } \\
\text { la tierra y cultivo del maíz. } \\
\text { 3.3. Símbolo de purificación, debido } \\
\text { a su capacidad de transmutar el alma } \\
\text { oscura por una nueva } \\
\text { 3.4. Símbolo de temor, dada su } \\
\text { asociación con la muerte, brujería y } \\
\text { hechicería. } \\
\text { 3.5. Como elemento de conexión entre } \\
\text { el mundo espiritual y terrenal. }\end{array}$ & $\begin{array}{l}\text { El carácter dual del murciélago genera } \\
\text { en la colectividad temor pero a la vez } \\
\text { admiración, por lo que se evita causarle } \\
\text { daño a la vez que se protegen las áreas } \\
\text { de vegetación aledañas a las cuevas, } \\
\text { favoreciendo de modo indirecto la } \\
\text { conservación del hábitat y de otras } \\
\text { especies animales que cohabitan en } \\
\text { estas áreas forestales. }\end{array}$ \\
\hline 4. Mítico & $\begin{array}{l}\text { 4.1. Relato que evoca el origen } \\
\text { deificatorio del murciélago (Códice } \\
\text { Magliabechiano). } \\
\text { 4.2. Relato que ubica el papel del } \\
\text { murciélago como emisario divino. } \\
\text { 4.3. Relato que refiere el uso de las alas } \\
\text { del murciélago por otros animales para } \\
\text { poder volar y solicitar la lluvia a los } \\
\text { dioses (mitología yaqui). }\end{array}$ & $\begin{array}{l}\text { La tradición oral y grafica refuerza los } \\
\text { lazos de pertenencia a la naturaleza, } \\
\text { generando un vínculo de respeto por } \\
\text { las plantas, los animales, el agua y } \\
\text { la tierra, en el entendido de que las } \\
\text { comunidades humanas dependen de } \\
\text { estos recursos para satisfacer todas sus } \\
\text { necesidades materiales y culturales. }\end{array}$ \\
\hline
\end{tabular}


estado de Chiapas, se traduce como "lugar de murciélagos" o "junto a los murciélagos".

Cabe destacar que el murciélago fue el glifo emblema de la antigua ciudad maya de Copán en Honduras, e inclusive se le consideró un animal totémico (Schlesinger, 2001). En la cultura maya el murciélago también se representó en estelas, incensarios, vasijas y esculturas, inclusive formó parte de su calendario de 20 meses, cuyo glifo es el patrono del cuarto mes maya (Thompson, 1996).

La mayor parte de las representaciones prehispánicas de murciélagos sugieren la presencia de miembros de la familia Phyllostomidae, debido principalmente a la recreación de la gran hoja nasal que constituye una de las características más distintivas de esta familia de quirópteros. Dicha familia se encuentra en las regiones tropicales, subtropicales y templadas de América, algunas especies en su distribución llegan hasta el suroeste de Estados Unidos. La familia comprende 143 especies, de las cuales 55 viven en México (Ramírez et al., 2005). Aunque muchas especies son insectívoras, en el grupo encontramos especies frugívoras y nectarívoras, que pueden llegar a ser muy abundantes, como los géneros Artibeus, Carollia, Leptonycteris o Glossophaga, o las tres especies que llegan a alimentarse de sangre y que son conocidos por este hábito como murciélagos vampiros: Desmodus rotundus, que suele consumir la sangre de generalmente de diferentes especies de mamíferos, mientras que Diaemus youngi y Diphylla ecaudata se alimentan preferentemente de la sangre de diversas aves (Vaughan et al., 2000; Wetterer et al., 2000; Datzmann et al., 2010).

\section{Valores religiosos}

Los murciélagos han formado una parte importante de la mitología de las culturas del México prehispánico siendo una de las deidades más notables. Las pruebas en este caso se encuentran en las abundantes representaciones de estos animales en urnas de cerámica, esculturas, pinturas, estelas y códices antiguos y es posible apuntar que el principal valor religioso del murciélago fue su deificación. Al respecto se sabe que el culto de Camazotz empezó alrededor de 100 a. C. entre los zapotecas de Oaxaca (y posteriormente adoptado por los mayas Quiches), quienes veneraban a un monstruo antropomórfico con cuerpo de hombre y cabeza de murciélago asociado principalmente con la noche, la muerte y el sacrificio (Westhem, 2000).

En la región cultural mixteca-zapoteca del Estado de Oaxaca, el valor divinatorio del murciélago fue manifiesto, ya que se le asoció a Piquete Ziña (dios de la fertilidad), aunque ciertos autores señalan que puede representar el alter ego de Pitao cozobi, deidad del maíz y de la milpa. Entre los mayas, los quirópteros tuvieron una fuerte asociación con Camazotz deidad del inframundo cuya traducción puede referirse a "el murciélago del rayo de la muerte", "dios de las cavernas", "servidor de la muerte" o "gobernante del crepúsculo" (Melton, 1999). En el Popol Vuh el culto a esta deidad se manifiesta en un pasaje en el cual se narra que en el cuarto nivel del Xibalbá (semejante al inframundo de los aztecas), denominado Zotziha (casa de murciélagos), habita Camazotz y miles de murciélagos que revolotean y chillan intermitentemente (Rencinos, 1995).

Entre los mexicas, el murciélago también es considerado ser del inframundo y se le asoció a la decapitación, ideología que trascendió y se incorporó al pensamiento religioso entre los pueblos nahuas. Se relacionaron con Tlacatzinacantli, deidad de la noche y el inframundo considerado como el murciélago arrancador de cabezas tal y como se constata en los códices Borgia, Vaticano y Féjérváry-Mayer (Aguilera, 1985; Seler 1980, 1996). También se postula que la figura de este mamífero alado también puede tener un valor como alter ego zoomorfo o nahualtin de algunas divinidades mesoamericanas, que como Quetzalcóatl y Tezcatlipoca podía tener varios dobles animales (Pérez, 1997; González, 2001). Asimismo, el murciélago se vincula a deidades como Xochipilli y Mictlantecuhtli, destacando su advocación con el inframundo (Figura 1), el mundo terrestre y el mundo celeste; por lo que es uno de los animales mesoamericanos que puede conectarse entre los tres niveles (Oliver, 1999). 
La sacralización del murciélago es un valor asociado a los templos nahuas conocidos como Cuauhcalli o "Casa del Sol", los cuales disponían de varias cámaras. Una de éstas cámaras era conocida como Tzinacalli (casa del murciélago) compuesta por dos cuartos, uno semicircular y el otro rectangular. Se arguye que en el Tzinacalli tenían lugar los rituales de iniciación de los guerreros Ocelotl (jaguar) y Cuauhtli (águila) (Weor, 2005). Se ha señalado que sobre la puerta de acceso del primer cuarto rectangular se colgaba un bloque de obsidiana y luego se encendía leña en la oquedad del suelo, y con la luz de la fogata el candidato se paraba frente al espejo de obsidiana para someterse al proceso de purificación espiritual que consistía en soportar el embate psíco-alucinógeno de Tlacatzinacantli, si resistía el tiempo necesario, pasaba al cuarto semicircular mientras un sacerdote incineraba la efigie del candidato hecha en papel de amate, simbolizando así el paso de las tinieblas a la luz, pues el alma animal de estos iniciados era como murciélago; es decir, estaba ciega y privada de poder por la falta de luz espiritual (Townsend, 1982; Noguez, 2006).

\section{Valores simbólicos}

Esta categoría comprende básicamente el simbolismo de los quirópteros asociado a la fertilidad femenina y de la tierra. En este caso, de acuerdo con el relato azteca relacionado con la siembra señala que: los dioses (al parecer
Quetzalcóatl y Tezcatlipoca) encomendaron al murciélago que le cortara a Xochiquétzal la flor que tenia adentro de su órgano sexual. Dicha empresa fue cumplida por el murciélago, el cual llevó la flor a los dioses quienes la lavaron y de esas aguas se produjeron unas flores con mal aroma, por lo que se le encomendó al murciélago que llevara esas flores al Mictlan en donde al ser lavadas nuevamente renacieron como flores aromáticas llamadas súchiles (Benítez, 1968). Esta escisión figurada simboliza la consagración de la diosa como mujer a la vez que se vincula a la menstruación y por lo tanto a la capacidad femenina de procrear (Johansson, 2000; Muñoz, 2006).

Entre los mixtecos y zapotecos, el murciélago también fue símbolo de fertilidad de la tierra, ya que era la conexión entre el suelo donde se siembra el maíz y el agua que lo fertiliza y le permite crecer. Por lo que el culto a este mamífero está ampliamente representado en esta región cultural en dinteles, vasos, silbatos, braseros y urnas, entre esta última categoría destaca una urna en la cual el dios murciélago presenta unas orejeras en forma de mazorcas de maíz (Westhem, 2000; Padilla, 2001; Ávila, 2002).

Cabe señalar que en nuestros días entre los indígenas del estado de Guerrero, México, persiste el uso de máscaras con diseños estilizados de murciélago (Figura 2), cuyo simbolismo purificatorio es utilizado en una ceremonia religiosa en la cual se cubren la cara con la

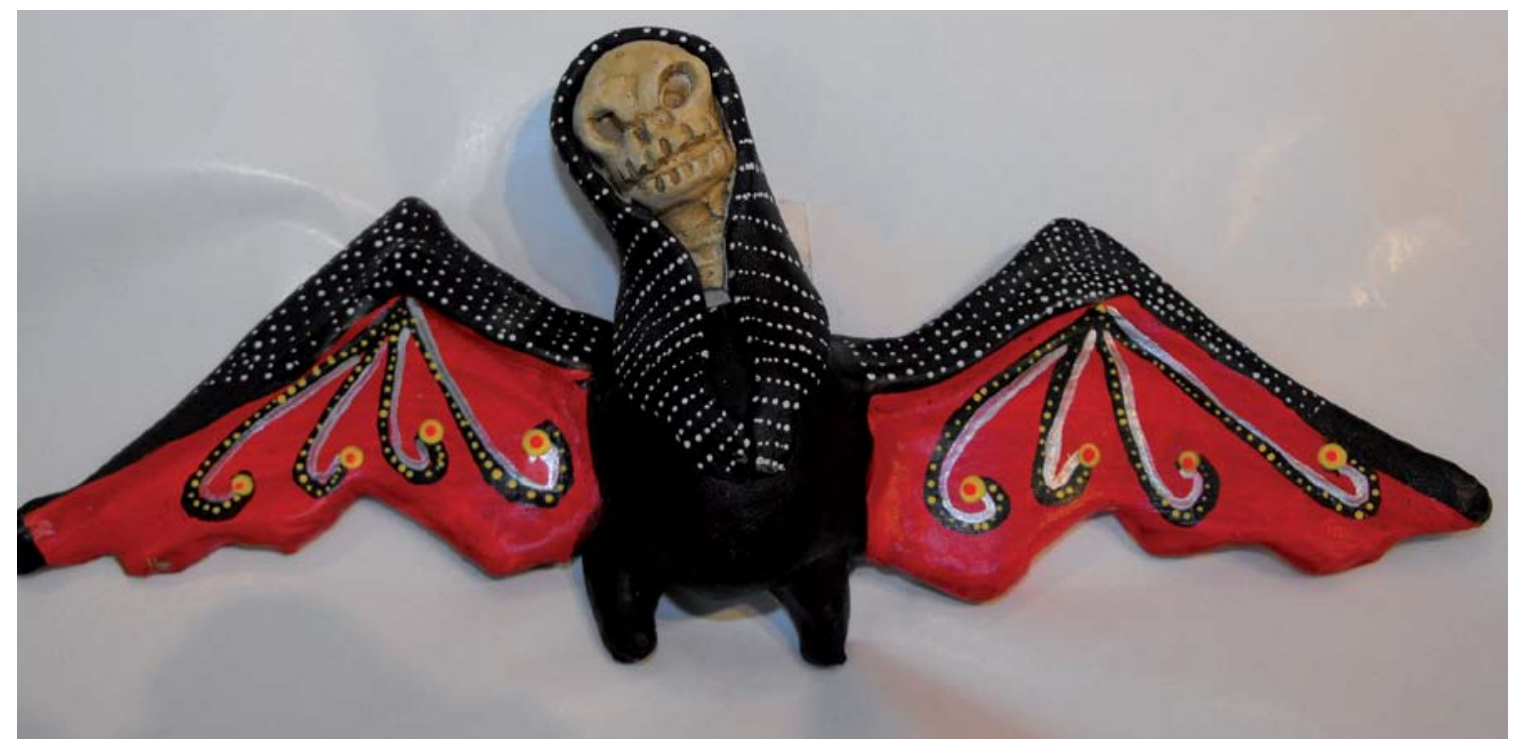

Figura 1. Figura de murciélago en barro elaborada en el estado de Oaxaca como motivo día de muertos, enmarcando la advocación con el inframundo y la muerte. 
Figura 2. Máscara con diseño de murciélago elaborada en el estado de Guerrero para uso ceremonial. Foto: R. Retana. máscara para hacer una transformación mística; es decir, una purificación al sustituir la antigua cara o alma por una nueva (Cordry, 2001; Charro, 1999).

\section{Valores míticos}

Durante el período prehispánico el origen de los murciélagos se explicó en un contexto divino, tal y como se reporta en el Códice Magliabechiano, en el cual se relata que Quetzalcóatl en uno de sus rituales expelió semen sobre una piedra y de allí nació el murciélago para servir de emisario divino, entre cuyas odiseas como se menciono anteriormente se narra el viaje que hizo para cercenarle de un bocado el órgano sexual a Xochiquétzal mientras dormía (Johansson, 2000; Muñoz, 2006).

Actualmente el valor mítico del murciélago aun persiste entre los Yaquis de Sonora en un antiguo relato popular explican que en los tiempos pasados los líderes de los ocho pueblos se reunieron en el Vícam y le pidieron al sapo Bobok que fuera con Yuku, dios de la lluvia, y

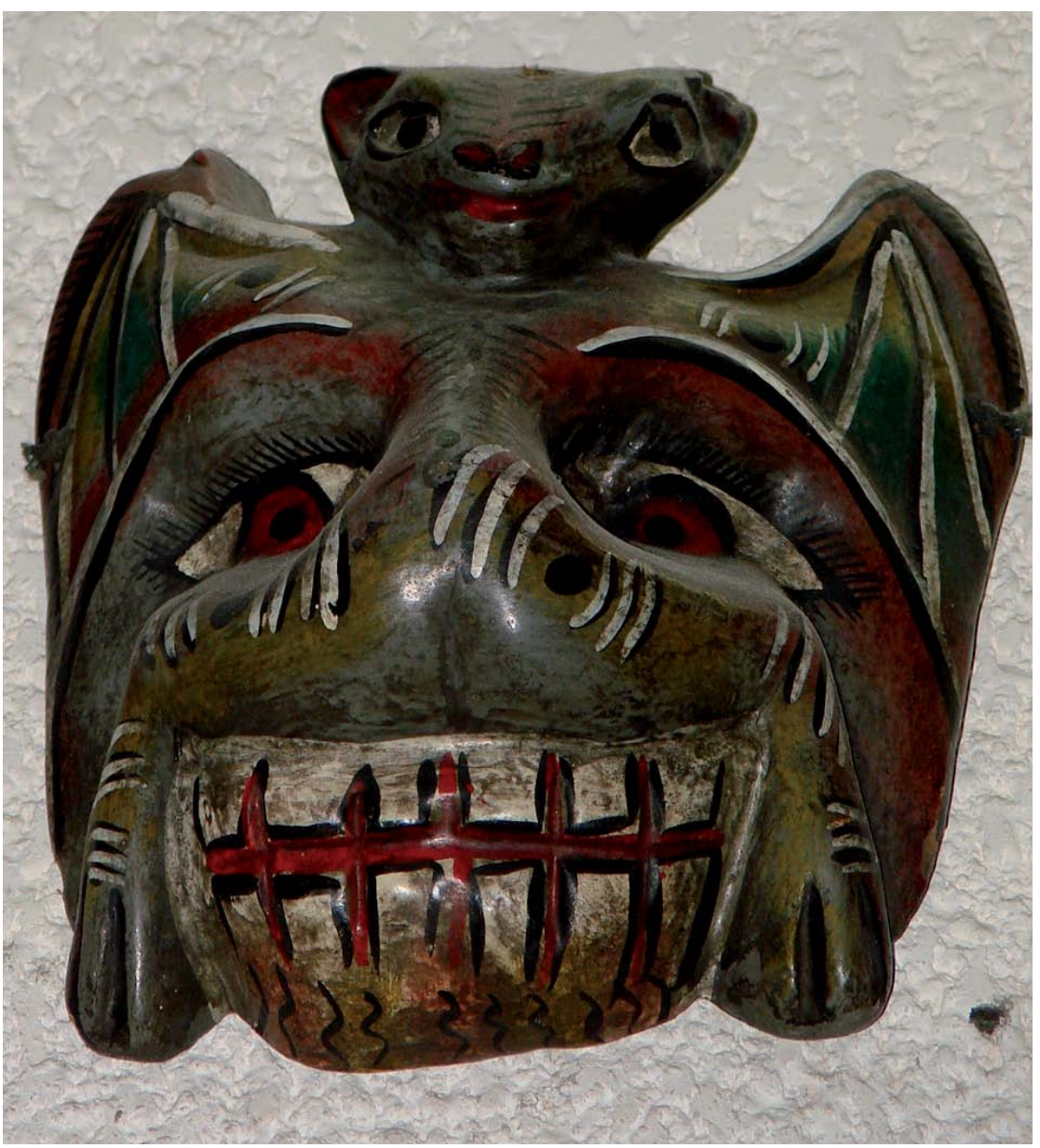

le pidieran mandase agua. El sapo se fue a la laguna y ahí un amigo que era mago le dio alas de murciélago con las cuales pudo volar hacia las nubes y encontrar a Yuku.

\section{DISCUSIÓN}

En el proceso de conocimiento y uso de la biodiversidad por parte de una sociedad humana se produce de manera intrínseca un vínculo y asignación de valores culturales a ciertas especies vegetales y animales (Navarijo, 1999). Estos valores culturales se definen como construcciones que operan en la conciencia colectiva y se articulan con todos los procesos vitales y espirituales del grupo humano que los genera. Son producciones locales y pueden ser de muy diversa índole y carácter; por ejemplo, con un significado emotivo, identificador, estético, simbólico y espiritual (Hunn, 1999; Schwartz, 2001). En este contexto, se dispone de diversos conocimientos biológicos para establecer que la atribución de valores culturales a los murciélagos u otros animales es consecuencia, en primera instancia, del reconocimiento de ciertas cualidades anatómicas, locomotoras y etológicas por parte del grupo social que las incorpora a su cosmovisión; trasmite como vínculos de poder, ascetismo, temor, fortuna, adoración, magia, y cuando una de estas relaciones se posiciona en el psique colectivo trasciende como un valor cultural (Schwartz, 2001).

En la medida que los valores culturales son asimilados y respetados por la colectividad, sea por su aspecto simbólico de sacralidad, prohibición, miedo a un dios castigador o por respeto a la palabra de los antepasados, estos valores comienzan a actuar como elementos de orden y control social, ya que permiten evaluar la bondad de las acciones individuales y colectivas hacia el entorno natural del cual dependen para satisfacer sus necesidades materiales y espirituales. Por lo tanto, los valores atribuidos a los murciélagos por las sociedades del México prehispánico actuaron de manera directa o indirecta como agentes reguladores en los procesos del conocimiento y uso de la fauna silvestre y su hábitat; es decir, formaron parte de las estrategias locales de conservación de los recursos del entorno natural. 
Bajo la perspectiva anterior, se ha reconocido y destacado a nivel nacional en la Estrategia Nacional de Biodiversidad e internacionalmente en los convenios de Diversidad Biológica, Cambio Climático, contra la Desertificación, y Desarrollo Sostenible, así como en los objetivos de Desarrollo del Milenio de las Naciones Unidas y en la Evaluación de los Ecosistemas del Milenio, que los valores culturales constituyen un elemento importante de gestión local respecto al uso de los recursos naturales presentes en los territorios comunales, por lo que deben ser considerados en la elaboración y ejecución de los programas relativos al uso sostenible del patrimonio natural, planteando la necesidad de integrar los modelos científicos con el propósito de asegurar prácticas que garanticen la conservación de la diversidad biológica (Millennium Ecosystem Assessment, 2005).

En el contexto anterior, la Convención Relativa a los Humedales de Importancia Internacional (RAMSAR, 2005), ha ubicado la trascendencia de considerar los valores culturales en sus estrategias de conservación y uso sostenible de los mismos. Por lo que recomienda incluir seriamente estos valores ya que pueden facilitar el fortalecimiento o restablecimiento de vínculos beneficiosos entre las personas y este tipo de vegetación, además de constituir una importante herramienta en la gestión de otros sistemas ecológicos. En este sentido, es necesario que en los proyectos de uso y conservación de la fauna silvestre en el medio rural, los investigadores consideren los valores culturales en la integración de los planes de manejo de este recurso, con la finalidad de evitar la generación de conflictos locales y/o regionales. (Pandey, 1997).

\section{CONCLUSIONES}

Los valores culturales vinculados a los murciélagos durante el México prehispánico, son producto en primera instancia de un proceso de conocimiento de este grupo animal, comprendiendo diversos aspectos de su morfología, etología y biología. Por lo que el reconocimiento de estos valores puede constituir un índice y referencia primaria de las relaciones y vínculos de una sociedad con su entorno natural. En este sentido, la identificación actual de los valores culturales otorgados a la fauna silvestre entre las comunidades indígenas y/o campesinas que habitan hoy día en México, pueden ser aplicados en el desarrollo de estrategias comunitarias sustentables que favorezcan los esfuerzos de conservación en torno a los murciélagos y a la biodiversidad en general.

Sabemos que a nivel mundial los murciélagos son el segundo orden de la clase Mammalia con el mayor número de especies, son los únicos mamíferos capaces de volar y dominar el espacio de la noche, forma de vida que les valió un lugar en las manifestaciones culturales de distintas sociedades humanas. En México, tras la conquista y la misión evangelizadora, los valores culturales vinculados a los murciélagos se fueron sustituyendo paulatinamente al grado de llegar a asociar el murciélago a seres demoníacos o símbolo de lo maléfico, causando principalmente entre las poblaciones de las ciudades una actitud de temor hacia este grupo de mamíferos al grado de considerárseles una amenaza. Afortunadamente, esta concepción esta cambiando al revalorizarse cada vez más su verdadera importancia ecológica y cultural a través de su difusión en distintos niveles de la sociedad.

\section{Agradecimientos}

Externamos nuestro agradecimiento a C. Lorenzo por la revisión y observaciones recomendadas a este manuscrito.

\section{Literatura citada}

Aguilera, C. 1985. Flora y Fauna Mexicana: Mitología y Tradiciones. Editorial Everest Mexicana, S. A. Ciudad de México, México.

Ávila, R. 2002. Los pueblos mesoamericanos. Instituto Politécnico Nacional. Ciudad de México, México.

Benítez, F. 1968. Los indios de México. Tomo II. Ediciones Era, S. A. de C. V. Ciudad de México, México.

Castro, A., y J. Galindo. 2009. Murciélagos en el México de ayer y hoy. Revista de Divulgación Científica y Tecnológica de la Universidad Veracruzana, 22:53-56.

Ceballos, G., J. Arroyo, R.A. Medellín y Y. Domínguez. 2005. Lista Actualizada de los Mamíferos de México. Revista Mexicana de Mastozoología, 9: 21-71.

Charro, M. 1999. Murciélagos: Príncipes de las Tinieblas. Revista Folklore,19:111-118. 
Cordry, D. 2001. Mexican Masks. University of Texas Press, Austin, Texas.

Datzmann, T., O. von Helversen and F. Mayer. 2010. Evolution of nectarivory in phyllostomid bats (Phyllostomidae Gray, 1825, Chiroptera: Mammalia), BMC Evolutionary Biology, 10:165.

Eguiarte, L. 2006. Los murciélagos en México. Arqueología Mexicana, 14(80):24-25.

González, Y. 2001. Animales y plantas en la cosmovisión mesoamericana. Plaza y Valdez, S. A. de C. V. Ciudad de México, México.

Grube, N. 2005. Toponyms, Emblem Glyphs, and the Political Geography of Southern Campeche. Anthropological Notebooks, 11:89102.

Hunn, E. 1999. El valor de la subsistencia para el futuro del mundo. Pp. 65-74, en: La Etnobiología en México: Reflexiones y Experiencias (Vásquez, M. ed.). Secretaría de Educación Pública-Consejo Nacional de Ciencia y Tecnología-Delegación Regional Sur. Ciudad de México, México.

Johansson, K. 2000. Escatología y muerte en el mundo náhuatl precolombino. Estudios de Cultura Náhuatl, 31:149-183.

La Duke, W. 1994. Traditional Ecological Knowledge and Environmental Futures. Journal of International Environmental Law and Policy, 5:127-148.

Macazaga, C. 1979. Nombres geográficos de México. Editorial Cosmos, Ciudad de México, México.

Medellín, R.A., H.T. Arita, y O. Sánchez. 2008. Identificación de los murciélagos de México: Clave de campo. Segunda edición. Instituto de Ecología, Universidad Nacional Autónoma de México - Comisión para el Conocimiento y Uso de la Biodiversidad.

Melton, J. G. 1999. The Vampire Book: The Encyclopedia of the Undead. Detroit, Visible Ink Press.

Millennium Ecosystem Assessment. 2005. Ecosystems And Human Well-Being: Biodiversity Synthesis. World Resources Institute, Washington, D. C

Muñoz, M. 2006. El culto al dios Murciélago en Mesoamérica. Arqueología Mexicana, 14(80):17-23.
Navarijo, L. 1999. Las aves como objetos culturales. Pp. 87-99, en: La Etnobiología en México: Reflexiones y Experiencias (Vásquez, M. ed.). Secretaría de Educación Pública-Consejo Nacional de Ciencia y Tecnología-Delegación Regional Sur. Ciudad de México, México.

Noguez, X. 2006. El Templo Monolítico de Malinalco, Estado de México. Arqueología Mexicana, 78:68-73.

Oliver, G. 1999. Los animales en el mundo prehispánico. Arqueología Mexicana, 6:4-15.

Padilla, A. 2001. Personaje de mitos, símbolo calendárico, deidad en los códices. El correo del Maestro, 65:1-9.

Pandey, D. 1997. Etnhoforestry by Indigenous People. Documento del XI Congreso Forestal Mundial. Antalya, Turquia.

Pérez, T. 1997. Los Olmecas y los Dioses del Maíz en Mesoamérica. Pp. 17-58, en: De Hombres y Dioses (Noguez, X. y A. López, coords.). El Colegio de Michoacán, México.

Ramírez, P.J., J. Arroyo y A. Castro. 2005. Estado Actual y Relación Nomenclatural de los Mamíferos Terrestres de México. Acta Zoológica Mexicana (n.s.), 21:21-82.

RAMSAR. 2005. Resolución IX.21. Tomar en cuenta los valores culturales de los humedales. $9^{a}$ Reunión de la Conferencia de las Partes Contratantes de la Convención Relativa a los Humedales de Importancia Internacional, Kampala, Uganda.

Rencinos, A. 1995. Popol Vuh. Las antiguas historias del Quiché. Fondo de Cultura Económica. Ciudad de México, México.

Retana, O. 2006. Fauna Silvestre de México: Aspectos Históricos de su Gestión y Conservación. Fondo de Cultura Económica. Ciudad de México, México.

Sarukhán, J., y R. Dirzo (comps). 1992. México ante los retos de la Biodiversidad. Comisión para el Conocimiento y Uso de la Biodiversidad. Ciudad de México, México, D.F.

Schlesinger, V. 2001. Animals and Plants of the Ancient Maya. University of Texas Press. Austin, Texas.

Schwartz, S. 2001. Value hierarchies across cultures. Talking a similarities perspective. Journal of Cross-Cultural Psychology, 32:268-290.
Seler, E. 1980. Comentarios al Códice Borgia. Fondo de Cultura Económica. Ciudad de México, México.

Seler, E. 1996. The Animal Pictures of the Mexican and Maya Manuscripts. Pp. 167-340, en: Collected Works in Mesoamerican Linguistics and Archaeology (Bowditch, C. ed.). Vol. 5. Labyrinthos, Culver City California.

Simmons, N. B. 2005. Orden Chiroptera. Pp. 312-529, en: Mammal species of the world. A taxonomic and geographic reference (Wilson, D. E., y D. M. Reeder, eds.). The Johns Hopkins University Press, Baltimore, EE.UU.

Tenorio, J. 1988. Técnicas de Investigación Documental. Mac Graw- Hill. Ciudad de México, México.

Thompson, E. 1996. Maya Hieroglyphs of the Bat as Metaphorgrams. Man New Series, 2(2):176-184

Toledo, V. 1991. Patzcuaro's Lessons: Nature, Production and Culture in an Indigenous Region of Mexico. Pp. 147-171, en: Biodiversity: Culture, Conservation, and Ecodevelopment (Olfield, M. y J. Alcorn, eds.). Westview Press. Boullder.

Townsend, R. 1982. Malinalco and the Lords of Tenochtitlan. Pp. 119-120, en: The Art and Iconography of Late Post-Classic Central Mexico (Boone, E. ed.). Dumbarton Oaks, Washington, D.C

UNESCO. 1999. Declaración de Noumea sobre la protección del saber tradicional. Organización de las Naciones Unidas para la Educación la Ciencia y la Cultura Noumea, Nueva Caledonia.

Vaughan, T. A., J. M. Ryan, N. J. Czaplewski. 2000. Mammalogy. Fourth Edition. Saunders College Publishing, Philadelphia

Vega, C. 1991. Códice Azoyu 1, el reino de Tlachinollan. Fondo de Cultura Económica. Ciudad de México, México.

Weor, S. 2005. Aztec Chritic Magic. Gnostic Kabbalah and Tarot in the American Mysteries. Glorian Publishing.

Westhem, P. 2000. Obras Maestras del México Antiguo. Siglo XXI Editores S. A. de C. V. Ciudad de México, México.

Wetterer, A., M. Rockman y N. Simmons. 2000. Phylogeny of Phyllostomid Bats (Mam- 
malia: Chiroptera): data from diverse morphological systems, sex chromosomes, and restriction sites. Bulletin of the American Museum of Natural History, 248: 1-200.
Wilson, D.E. y D.M. Reeder (eds.). 2005. Mammal Species of the World. The Johns Hopkins University Press, Baltimore, EE.UU. 